\title{
Antibiotic Sensitivity Patterns in Children with Urinary Tract Infection: Retrospective Study Over 8 Years in a Single Center
}

\author{
ByungwooWoo, M.D. \\ Youngkwon Jung, M.D. \\ Hae Sook Kim, M.D. \\ Department of Pediatrics, Daegu \\ Fatima Hospital, Daegu, Korea
}

Corresponding author: Hae Sook Kim, M.D. Department of Pediatrics, Daegu Fatima Hospital, 99, Ayang-ro, Dong-gu, 41199,

Daegu, , Korea

Tel: +82-53-940-7243

Fax: +82-53-940-7076

E-mail: coreroo@hanmail.net

Received: 23 February 2018

Revised: 27 March 2018

Accepted: 19 June 2018
Purpose: We studied the pathogens and trends in antibiotic sensitivity pattern in children with urinary tract infection (UTI) over 8 years in order to evaluate adequate treatment.

Methods: We performed a retrospective review of medical records of children with UTI from January 2009 to December 2016 in Daegu Fatima Hospital. Uropathogens and antibiotic sensitivity patterns were selected. Only 1 bacterial species with a colony count of $\geq 105 \mathrm{CFU} / \mathrm{mL}$ was considered a positive result. We compared 2 periods group (A: 2009 2012, B: 2013 2016) to investigate trends of antibiotic sensitivity pattern.

Results: During the 8 year period, 589 cases are identified (E. coli was cultured in 509 cases, 86.4\%). Among all patients, this study investigated the antibiotic sensitivity of $E$. coli. Antimicrobial susceptibility to ampicillin was steadily low for both periods (A: $32.6 \%, B: 40.1 \%, P=0.125$ ), and to amikacin was consistently high for both periods (A: $99.4 \%, B: 99.3 \%, P=1.000$ ). Antibiotic sensitivity to third-generation cephalosporin decreased from period $A$ to $B(A: 91.7 \%, B: 75.5 \%, P=0.000)$. Antibiotic sensitivity to quinolone significantly decreased from $A$ to $B$ (A: $88.4 \%$, $B$ : $78.2 \%, P=0.003)$. The prevalence of extended-spectrum $\beta$-lactamase-producing E. coli increased from period A to B (A: $6.1 \%, B: 17.1 \%, P=0.000$ ).

Conclusion: This study showed that conventional antibiotic therapy for the treatment of pediatric UTI needs to be reevaluated. A careful choice of antibiotic is required due to the change in antibiotic sensitivity and the emergence of antibiotic-resistant bacteria.

Key words: Urinary tract infection, antibiotic sensitivity, Extended-spectrum $\beta$-I actamase

\section{Introduction}

Urinary tract infection (UTI) is one of the most common forms of pediatric

This is an open-access article distributed under the terms of the Creative Commons Attribution Non-Commercial License (http:// creativecommons.org/licenses/by-nc/4.0/) which permits unrestricted non-commercial use, distribution, and reproduction in any medium, provided the original work is properly cited. bacterial infections and is the second most common infectious disease in children following respiratory infections ${ }^{1)}$. UTI in children manifests as nonspecific symptoms such as fever with uncertain infectious site, which is the main reason for antibiotic treatment upon visiting the emergency room or hospitalization ${ }^{2}$. The prognosis of UTI is good, but long-term complications can lead to renal insufficiency, hypertension, and chronic kidney failure if patients have anatomical abnormalities or proper treatment is not initiated ${ }^{2,3}$. 
For this reason, children with suspected UTI should be started on empirical antibiotic therapy before urine culture results are confirmed, to prevent renal impairment. According to sex and age, the incidence and clinical symptoms vary, and the causative organisms are different in pediatric UTI. In addition, as antibiotic-resistant pathogens change and are increased due to the abuse of antibiotics and inappropriate selection, it is necessary to select proper antibiotics in the early phase of treatment. Thus, regular antibiotic sensitivity results from the evaluation of UTI are important to improve the effectiveness of treatment.

We performed this study to help in the selection of appropriate antibiotics by investigating the trend of causative bacteria, antibiotic susceptibility and the frequency of extended-spectrum $\beta$-lactamase (ESBL)-producing bacteria in 589 patients with UTI who were admitted to Daegu Fatima Hospital within a period of 8 years.

\section{Materials and methods}

\section{Patient selection}

We retrospectively reviewed the cases of children aged 0 to 12 years who were hospitalized for febrile UTI at the Department of Pediatrics, Daegu Fatima Hospital, from January 2009 to December 2016. We defined febrile UTI as a case of fever with a tympanic temperature of $38^{\circ} \mathrm{Cor}$ more, 5 or more white blood cells per high power field on urine sediment microscopy, and more than $10^{5}$ colony forming units $(\mathrm{CFU}) / \mathrm{mL}$ on urine culture analysis. Only 1 bacterial species identified with a colony count of $\geq 10^{5}$ $\mathrm{CFU} / \mathrm{mL}$ was considered a positive result (when two or more strains were cultured, they were excluded). Among toilet-trained children, midstream urine was collected, whereas among children who are not toilet trained, urine was collected by an adhesive sterile collection bag after sterilization of the skin of the genitals.

The distribution of sex and age, distribution of major pathogens, antibiotic sensitivity and antibiotic sensitivity of ESBL-producing E. coli were retrospectively analyzed in 589 patients with UTI.

\section{Organism identification and drug susceptibility test}

The identification of cultured strains and antibiotic susceptibility tests were performed based on the Clinical and Laboratory Standards Institute guidelines. Cultures were performed using $5 \%$ sheep blood agar plate and MacConkey agar plate, which were inoculated with $0.001 \mathrm{~mL}$ of urine each, and incubated at $36^{\circ} \mathrm{C}$ in $5 \% \mathrm{CO}_{2}$ for 48 hours. The formed colonies after culturing were identified by biochemical analysis and those containing more than $10^{5} / \mathrm{mL}$ cultures were judged to be causative organisms of UTI. The strains isolated from urine culture samples were tested for susceptibility to antibiotics used in clinical trials. In our study, we used Microscan ${ }^{\circledR}$ (Dade Behring, Milton Keynes, $\mathrm{UK})$, which is an automated instrument, to assess bacterial identification and antibiotic susceptibility.

\section{Statistical analysis}

Statistical analysis was performed using SPSS version 18.0 (IBM Corp., Armonk, NY, USA). Chi-square test or Fisher's exact test (less than 5 values) was used for the analysis of categorical variables, and Chi-square test for trend was used to evaluate the changes according to periods. The mean values of some variables were compared between the two groups using the student's t-test. A $P$ value less than 0.05 was considered statistically significant.

\section{Results}

\section{Patient characteristics}

Among 589 patients diagnosed with UTI, 382 patients (64.8\%) were male, and 207 patients (35.2\%) were female; the male to female ratio was 1.8:1. The mean age of females was slightly higher than that of males: 5.6 months for males and 14.3 months for females. In the group younger than 1 year, the male to female ratio was 2.3:1, which showed a higher number of males (362 cases were males, and 157 cases were females). In the group aged 1 to 2 years, the male to female ratio was 1:5.4, which showed a higher number of females ( 5 cases were males, and 27 cases were females) (Table 1). 


\section{Trend of periodic antibiotic sensitivity}

We compared 2 periods groups (A: 2009-2012, B: 20132016) to investigate trends of antibiotic sensitivity patterns. During the 8 year period, 589 cases are collected (E. coli was cultured in 509 cases, $86.4 \%)$. Among all patients, this study investigated the antibiotic sensitivity of $E$. coli. The antimicrobial susceptibility to ampicillin was steadily low (A: $32.6 \%, \mathrm{~B}: 40.1 \%, P=0.125$ ), to amikacin was consistently high (A: $99.4 \%, \mathrm{~B}: 99.3 \%, P=1.000$ ), to trimethoprim/sulfamethoxazole was steadily moderate (A: 72.1\%, B: 70.7\%, $P=0.757)$, to carbapenem was steadily high (A: $99.7 \%$, B: $100 \%, P=1.000$ ), and to piperacillin/tazobactam was steadily high for 2 periods (A: $94.2 \%, \mathrm{~B}: 95.9 \%, P=0.460$ ). These changes were not statistically significant.

Antibiotic sensitivity to third-generation cephalosporin significantly decreased from period A to B (A: $91.7 \%, \mathrm{~B}$ : $75.5 \%, P=0.000)$. Antibiotic sensitivity to quinolone significantly decreased from A to B (A: $88.4 \%, \mathrm{~B}: 78.2 \%, P=$ 0.003). Antibiotic sensitivity to monobactam significantly decreased from period $\mathrm{A}$ to $\mathrm{B}$ (A: 91.4\%, B: 77.6\%, $P=0.000$ ).

Table 1. Age and Sex Distribution of Patients of All Pathogens and Escherichia coli

\begin{tabular}{lccccccc}
\hline \multirow{2}{*}{$\begin{array}{l}\text { Age } \\
\text { (yrs) }\end{array}$} & \multicolumn{3}{c}{ Total, N (\%) } & & \multicolumn{3}{c}{ E.coli, N (\%) } \\
\cline { 2 - 5 } \cline { 7 - 8 } & Male & Female & Total & & Male & Female & Total \\
\hline $0-1$ & $362(61.5)$ & $157(26.6)$ & $519(88.1)$ & & $324(63.7)$ & $131(25.7)$ & $455(89.4)$ \\
$1-2$ & $5(0.8)$ & $27(4.6)$ & $32(5.4)$ & & $4(0.8)$ & $19(3.7)$ & $23(4.5)$ \\
$>2$ & $15(2.5)$ & $23(4.0)$ & $386(6.5)$ & & $8(1.6)$ & $23(4.5)$ & $31(6.1)$ \\
Total & $382(64.8)$ & $207(35.2)$ & $589(100)$ & & $336(66.1)$ & $173(33.9)$ & $509(100)$ \\
\hline
\end{tabular}

Table 2. Comparison of Susceptibility of Escherichia coli to Antibiotics between 2 Periods

\begin{tabular}{lccc}
\hline \multirow{4}{*}{ Antibiotics } & \multicolumn{2}{c}{ No. (\%) of susceptible E. coli } & \\
\cline { 2 - 3 } & $\begin{array}{c}2009-2012 \text { (period A) } \\
(\mathrm{N}=362)\end{array}$ & $\begin{array}{c}2013-2016 \text { (period B) } \\
(\mathrm{N}=147)\end{array}$ & $P$ value \\
\hline AMP & $118(32.59)$ & $59(40.13)$ & 0.125 \\
AMK & $360(99.44)$ & $146(99.31)$ & 1.000 \\
TMP/SMX & $261(72.09)$ & $104(70.74)$ & 0.757 \\
CBP & $361(99.72)$ & $147(100)$ & 1.000 \\
TZP & $341(94.19)$ & $141(95.91)$ & 0.460 \\
3rd CEP & $332(91.70)$ & $111(75.51)$ & 0.000 \\
QNL & $320(88.39)$ & $115(78.23)$ & 0.003 \\
MNB & $361(91.43)$ & $114(77.55)$ & 0.000 \\
ESBL & $22(6.07)$ & $25(17.06)$ & 0.000 \\
\hline Abb & &
\end{tabular}

Abbreviations: AMP, ampicillin; AMK, amikacin; TMP/SMX, trimethoprim/ sulfamethoxazole; CBP, carbapenem; TZP, piperacillin/tazobactam; $3^{\text {rd }}$ CEP, third-generation cephalosporins; QNL, quinolone; MNB, monobactam; ESBL, extended-Spectrum $\beta$-lactamase.
The prevalence of ESBL-producing $E$. coli significantly increased from period A to B (A: $6.1 \%, \mathrm{~B}: 17.1 \%, P=0.000$ ) (Table 2) (Fig. 1).

\section{Comparison of two groups with or without sus- ceptibility to third-generation cephalosporin antibiotics}

When we compared patients showing susceptibility to third-generation cephalosporins with patients showing resistance, the rate of previous hospitalization was significantly lower among the patients who showed susceptibility (10.6\% vs. $22.7 \%, P=0.011)$. The rate of change of antibiotics after initial treatment failure was also significantly lower in the susceptible group compared to the resistant group $(9.5 \%$ vs. $21.2 \%, P=0.011)$. There were no significant differences between the two groups regarding fever duration or age (Table 3).

\section{Comparison between the ESBL-producing E. coli infection group and the non-ESBL-producing $E$. coliinfection group}

The ESBL-producing E. coli infection group had a significantly higher rate of previous hospitalization compared to the non-ESBL-producing E. coli infection group (24.4\% vs. $10.6 \%, P=0.005)$. The rate of change of antibiotics after initial treatment failure was also significantly higher in the

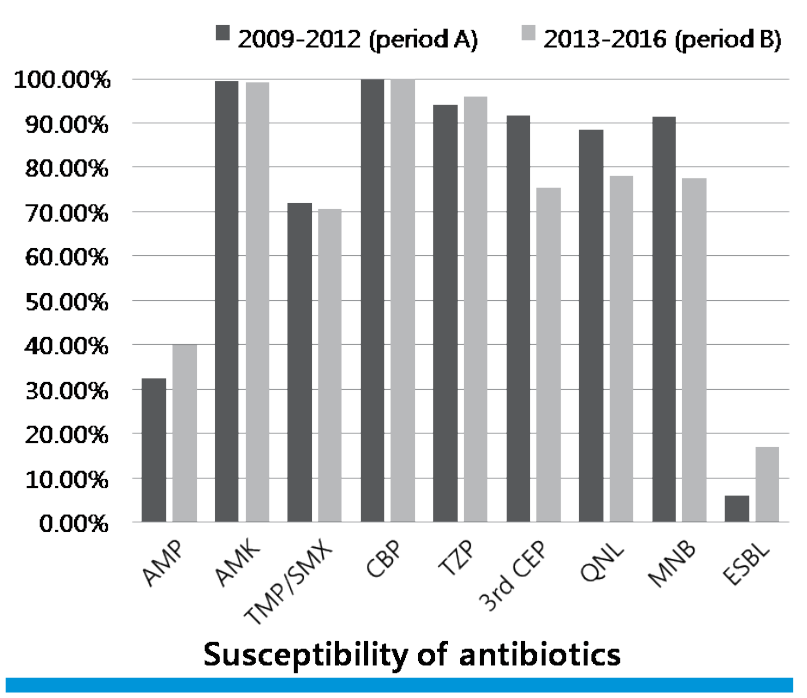

Fig. 1. Trend in antibiotic susceptibility of Escherichia. coli over 8 years. AMP, ampicillin; AMK, amikacin; TMP/SMX, trimethoprim/ sulfamethoxazole; CBP, carbapenem; TZP, piperacillin/tazobactam; $3^{\text {rd }}$ CEP, third-generation cephalosporins; QNL, quinolone; MNB, monobactam; ESBL, extended-spectrum $\beta$-lactamase. 
ESBL-producing $E$. coli group compared to the non-ESBLproducing $E$. coli group (22.2\% vs. 9.7\%, $P=0.005)$. There were no significant differences between the two groups regarding age, sex, fever duration, or blood tests (Table 4).

\section{Discussion}

The prevalence of pediatric UTI was $1 \%$ in males and $1-3$ $\%$ in females. Generally, males are diagnosed within the first year of life, while females are diagnosed at 3 years old. The male to female ratio of pediatric UTI patients varied according to age, from 2.8-5.4:1 within the first year of life and 1:10 between 1 and 2 years of age ${ }^{3)}$. In our study, the age at first diagnosis of UTI was higher in females than males, and the proportion of sex by age also showed a similar tendency. Because the proportion of children younger than 1 year who had a high prevalence rate in males was high, the

Table 3. Comparison of Clinical Presentations according to Susceptibility to Third-generation Cephalosporins

\begin{tabular}{lccc}
\hline & \multicolumn{2}{c}{ No. of episodes(\%) } & \\
\cline { 2 - 3 } & $\begin{array}{c}3 r d \text { CEP(+) } \\
(\mathrm{N}=443)\end{array}$ & $\begin{array}{c}3 \text { rd CEP(-) } \\
(\mathrm{N}=66)\end{array}$ & P value \\
\hline Age(months) & $7.85 \pm 100.15$ & $13.28 \pm 103.72$ & 0.081 \\
Fever duration(day) & $3.37 \pm 6.63$ & $3.58 \pm 6.42$ & 0.264 \\
$\begin{array}{l}\text { Change of antibiotics } \\
\text { during treatment }\end{array}$ & $42(9.5)$ & $14(21.2)$ & 0.011 \\
$\begin{array}{l}\text { Hospitalization within } \\
\text { previous 3 mon }\end{array}$ & $47(10.6)$ & $15(22.7)$ & 0.011 \\
\hline
\end{tabular}

Abbreviations: $3^{\text {rd }}$ CEP : third-generation cephalosporin. overall ratio of male and female patients with UTI showed opposite results in this study.

It is known that $80-90 \%$ of pediatric UTIs are caused by E. coli, and 10-15\% are caused by Klebsiella, Enterobacter, Proteus, and Pseudomonas ${ }^{1,3)}$. Enterococci, Pseudomonas, S. aureus, S. epidermidis, Haemophilus influenzae, Group $B$ streptococci can be cultured in children who have urinary tract abnormalities or dysfunction and Lactobacillus species, Corynebacterium species, Coagulase-negative staphylococci, and $\alpha$-hemolytic streptococci can be cultured when the urine is contaminated during collection ${ }^{4}$. The percentage distribution of causative organisms in urine cultures was similar to previous studies; E. coli (86.4\%) was the commonest pathogen, followed by Klebsiella (4.2\%). In addition, Enterococcus, Enterobacter, Proteus, Citrobacter, and Staphylococcus were major causative

As the number of antibiotic-resistant strains has been rapidly increasing, it is more difficult to treat patients with UTI. Because pediatric patients are more likely to have high morbidity and mortality when infected by resistant bacteria and the use of non-susceptible antibiotic may confer a poor prognosis, it is essential to select appropriate empirical antibiotics after considering the antibiotic susceptibility patterns ${ }^{5}$.

Thus, we investigated the antibiotic susceptibility patterns of $E$. coli that was the most common cause of UTI. Antimicrobial susceptibility to ampicillin was steadily low in both periods A and B. E. coli has two strains, 29A and 29B, of which 29A was resistant to ampicillin but showed a

Table 4. Comparison of Laboratory Findings and Clinical Characteristics between the ESBL-producing and the non-ESBL-Producing Escherichia coli Infection Groups

\begin{tabular}{|c|c|c|c|}
\hline & \multicolumn{2}{|c|}{ No. of episodes(\%) } & \multirow{2}{*}{$P$ value } \\
\hline & $\mathrm{ESBL}(\mathrm{N}=45)$ & Non-ESBL $(\mathrm{N}=464)$ & \\
\hline Age (months) & $14.24 \pm 102.76$ & $7.85 \pm 100.15$ & 0.074 \\
\hline Fever duration (day) & $2.69 \pm 5.31$ & $2.36 \pm 5.64$ & 0.109 \\
\hline WBC (cell//mm $\mathrm{mm}^{3}$ & $14,760.2 \pm 10,869.80$ & $13,638.81 \pm 22,811.19$ & 0.153 \\
\hline $\operatorname{CRP}(\mathrm{mg} / \mathrm{dL})$ & $6.51 \pm 22.46$ & $5.73 \pm 21.27$ & 0.37 \\
\hline ESR (mm/hr) & $14.71 \pm 68.29$ & $18.86 \pm 100.14$ & 0.245 \\
\hline Males younger than $1 \mathrm{yr}$ & $28(62.3)$ & $334(72.0)$ & 0.239 \\
\hline Females older than 1 yr & $7(15.6)$ & $43(9.3)$ & 0.134 \\
\hline Change of antibiotics during treatment & $10(22.2)$ & $45(9.7)$ & 0.005 \\
\hline Vesicoureteral reflux & $7(15.6)$ & $57(12.2)$ & 0.437 \\
\hline Hospitalization within previous 3 mon & $11(24.4)$ & 49 (10.6) & 0.005 \\
\hline Recurrence of UTI in 6 mon & $7(15.6)$ & $44(9.5)$ & 0.215 \\
\hline
\end{tabular}

Abbreviations: WBC, white blood cell; CRP, C-reactive protein; ESR, erythrocyte sedimentation rate; ESBL, extended-Spectrum $\beta$-lactamase. 
higher level of resistance after receiving ampicillin, and 29B was previously susceptible to ampicillin but showed resistance after ampicillin administration. It is believed that susceptibility to ampicillin, one of the most common antibiotics that can be prescribed from the newborn period, was steadily low due to this mechanism ${ }^{6}$.

The susceptibility of $E$. coli to trimethoprim/sulfamethoxazole (TMP/SMX) was moderate at $72.1 \%$ in period $\mathrm{A}$, and $70.7 \%$ in period $\mathrm{B}$, respectively. We concluded that TMP/SMX was not satisfactory as a primary antibiotic for the treatment of UTI. Lee et al. showed that the average resistance of $E$. coli to TMP/SMX was $34.3 \%^{7}{ }^{7}$. It has also been reported that susceptibility to TMP/SMX was 52.38 $\%$ (1995-2000), 50.00\% (2001-2005) and 48.1\% (2003-2005), $50.0 \%$ (2006-2008) in two other studies, respectively ${ }^{8,9)}$. Overall, susceptibility of $E$. coli to TMP/SMX was low in pediatric UTI patients, despite the variation of the results obtained in several studies. This is supported by the crossresistance mechanism, that is, resistance to trimethoprim also shows as resistance to TMP/SMX, ampicillin, ampicillin/clavulanate, and quinolone ${ }^{10}$.

Majority of study results showed that amikacin had a susceptibility higher than $90 \%$. In this study, amikacin also showed susceptibilities as high as $99.4 \%$ and $99.3 \%$ in both periods A and B, respectively. Amikacin should be used with careful consideration of complications such as ototoxicity and nephrotoxicity in children with UTI, and their serum creatinine levels should be constantly monitored.

Third-generation cephalosporin, which has shown high susceptibility in many studies and has been most commonly used in the treatment of pediatric UTI, also showed significantly reduced susceptibility in this study. The susceptibility was as high as $91.7 \%$ in period A, but significantly decreased to $75.5 \%$ in period B. Other studies also showed similar results. Jang et al.'s five-year study (1993-1997) identified that resistance of $E$. coli to ceftazidime increased from $3 \%$ to $14 \%{ }^{11}$. Lee et al. also determined that the resistance to third-generation cephalosporin increased to $2.1 \%$ in $2000-2004,8.3 \%$ in $2005-2009$, and $8.8 \%$ in $2010-$ 2014. This may be due to the gradually increasing resistance due to extensive use of third-generation cephalosporins for urinary tract, upper respiratory, and gastrointestinal infections. Even if the diagnosis is uncertain, the tendency to use antibiotics with a wide range of antimicrobial activity is also considered one of the causes ${ }^{7,12,13)}$. Another study reported that the continuous increase in the use of thirdgeneration cephalosporins has led to increased $E$. coli resistance (from $14 \%$ to $50 \%)^{14)}$. The results of our study showed that patients with resistance to third-generation cephalosporins were more likely to have been hospitalized in the preceding 3 months, and were also more likely to have changed antibiotics after initial treatment failure. These two differences were statistically significant, and it is thought that the same factors would have affected the B group, since they showed a higher detection rate of thirdgeneration cephalosporin-resistant bacteria in culture tests. Because we were unable to obtain medication histories for antibiotics prescribed outside our hospital, it was not possible to ascertain the precise frequency of previous antibiotic use. Thus, we postulated that the rate of antibiotic exposure would be higher among older patients, and investigated antibiotic susceptibility by age of diagnosis. We observed that patients showing third-generation cephalosporin susceptibility were 5 months younger, on average, than patients showing resistance, although this difference was not statistically significant. Thus, we expect that antibiotic medication history will also have an effect on susceptibility.

Quinolone is commonly used to treat cystitis in adults, but is rarely prescribed in children due to several side effects. In children, the use of quinolone has been approved only for cystic fibrosis, immunosuppression, severe typhoid fever, paratyphoid, and complicated urinary tract infection. However, studies using young animals showed that cartilage lesions were found in joints as a result of quinolone administration. Moreover, other reported side effects were increased QT interval on electrocardiogram, gastrointestinal damage, central nervous system damage, and photosensitivity, thereby requiring careful administration ${ }^{15)}$. In this study, E. coli's susceptibility to quinolone was significantly reduced from $88.4 \%$ to $78.2 \%$. Lee et al. reported that the resistance to quinolone increased to $7.9 \%$ in $2000-$ $2004,9.7 \%$ in $2005-2009$, and $12.4 \%$ in $2010-2014^{7)}$. A study found that drug resistance occurred through mechanisms that cannot be understood by similar drugs or other irrelevant drugs even without exposure. Another study argued that the conjugation of qnr (a plasmid-mediated quinolone gene, derived from other avirulent bacteria) into E. coli or K. pneumoniae (virulent bacteria) was 
an important mechanism of pediatric quinolone drug resistance development ${ }^{16}$. Therefore, quinolone abuse should be prevented in adults because it can cause resistance in children.

In recent years, the incidence of ESBL-producing gramnegative bacteria has increased, making it difficult to choose antibiotics. ESBLs are $\beta$-lactamases that hydrolyze cephalosporin and monobactams with an oxyimino side chain via a plasmid-mediated mechanism. Therefore, they have multi-resistance to antibiotics related to oxyimino- $\beta$ lactams ${ }^{17)}$. This resistance is mainly observed in $E$. coli and Klebsiella as well as other intestinal bacteria. It has been increasingly observed in bacteria, such as K. pneumoniae, Pseudomonas species, and E. coli worldwide. In Korea, ESBL-producing bacteria have been reported since the 1990s and have increased rapidly over the past decade. The incidence of ESBL in Korea was 4.8-7.5\% in Pai et al., study from 1994 to 1998, 10.8\% in Lee et al.'s study from 2001 to 2003, 11.03\% in Park et al., study from 2008 to 2010, and 13.6\% in Ahn et al.'s study from 2011 to 2013, indicating a continuous increase ${ }^{18-21)}$. In this study, a total of $45 \mathrm{ESBL}-$ producing strains were found, all of which were $E$. coli. The percentage of ESBL-producing strains was $6.1 \%$ in period $A$ and $17.1 \%$ in period $B$, thus showing a statistically significant increase in the incidence rate. In a study by Lee et al., the percentage of ESBL-producing strains continuously increased to $1.4 \%, 7.6 \%$, and $8.2 \%$ over the three periods ${ }^{7}$. Risk factors for increased ESBL-producing Gram-negative bacteria are decreased immunity, long-term hospitalization, and frequent use of cephalosporins due to the infection history, such as UTI. In addition, studies have shown that there were a significant number of infections caused by ESBL+ strains in male infants younger than 1-year-old and female infants older than 1-year-old. A study showed that risk factors included history of anatomical/functional urinary anomalies, recurrent urinary tract infections and sepsis, as well as hospital admission in the last 3 months ${ }^{22}$. In the results of our study, there were no significant differences between the ESBL-producing $E$. coli infection group and the non-ESBL-producing $E$. coli infection group regarding age, fever duration, or blood tests. However, the ESBLproducing group showed significantly higher rates of hospitalization in the preceding 3 months and change of antibiotics during treatment due to persistent fever. Also, the ESBL-producing E. coli group tended to show higher recurrence rates for vesicoureteral reflux and UTI, although this difference was not statistically significant. The recurrence rate and failure rate of UTI treatment due to ESBLproducing $E$. coli are high; therefore, the necessity of guidelines for antimicrobial therapy has developed. In this study, all of the children infected by ESBL+ strains were susceptible to carbapenem and $82 \%$ were susceptible to quinolone. Other studies also recommended carbapenem and quinolone. Therefore, these antibiotics should be used while carefully monitoring changes in antibiotic susceptibility.

This study has limitations because of the small sample size ( 589 for $>8$ years) with an uneven age distribution and was conducted in a limited local community. In addition, there was a large gap in the number of patients during the two comparative periods. Therefore, further studies should be conducted to overcome these limitations.

In conclusion, ampicillin and TMP/SMX, which are widely used as conventional antibiotics for treating pediatric UTI, may no longer be appropriate. Due to the decreased susceptibility to third-generation cephalosporin and quinolone depending on the period and continuously increasing ESBL strains, the discovery and use of appropriate antibiotics are required.

\section{Conflicts of Interest}

No potential conflict of interest relevant to this article was reported.

\section{References}

1. Brouhard BH, Travis LB. Infections of the urinary tract. In : Rudolph AM, editors. Pediatrics. 18th ed. Appleton \& Lange Co, 1987:1197202.

2. Hoberman A, Chao HP, Keller DM, Hickey R, Davis HW, Ellis D. Prevalence of urinary tract infection in febrile infants. J Pediatr 1993; 123:17-23.

3. Elder JS. Urinary tract infections. In: Kliegman RM, Stanton BF, St. Geme JW, Schor NF, Behrman RE, eds. Nelson textbook of pediatrics. 19th ed. Philadelphia, PA: Elsevier;2011:1829-834.

4. Fernbach SK, Maizels M, Conway JJ. Ultrasound garding of hydronephrosis: Introduction to the system used by the society for 
fetal urology. Pediatr Radiol 1993;23:478-80.

5. Kang Cl, Kim SH, Park WB, Lee KD, Kim HB, Kim EC,et al. Bloodstream infections caused by antibiotic-resistant gram-negative bacilli: risk factors for mortality and impact of inappropriate initial antimicrobial therapy on outcome. Antimicrob Agents Chemother 2005;49:760-6.

6. Karami N, Martner A, Enne VI, Swerkersson S, Adlerberth I, Wold AE. Transfer of an ampicillin resistance gene between two Escherichia coli strains in the bowel microbiota of an infant treated with antibiotics. J Antimicrob Chemother 2007;60:1142-5.

7. Lee YK, Lee HJ, Kim JM, Kang JM, Lee ST, Lee NY, Kim YJ, Cho HY. The Antibiotic Resistance Pattern of Gram-Negative Bacteria in Children Younger Than 24 Months with a Urinary Tract Infection: A Retrospective Single-Center Study over 15 Consecutive Years. Child Kidney Dis 2015;19:148-53.

8. Song YH, Kim DH, Park JY, Choi CH, Cho EY, Kim SM,et al. Escherichia coli susceptibility to antimicrobials in children with urinary tract infection. J Korean Soc Pediatr Nephrol 2006;10:192-200.

9. Ko JH, Lee JH, Shim EJ, Cho DJ, Min KS, Yoo KY, Lee DH, Kang HJ. Microbiological spectrum and antibiotic susceptibility pattern in more than 24-month-old children with urinary tract infection: A6-year retrospective, single center experience. Korean J Pediatr 2009:52:1147-52.

10. Zhanel GG, Hisanaga TL, Laing NM, DeCorby MR, Nichol KA, Weshnoweski B, Johnson J, Noreddin A, Low DE, Karlowsky JA; NAUTICA Group, Hoban DJ. Antibiotic resistance in Escherichia coli outpatient urinary isolates: final results from the North American Urinary Tract Infection Collaborative Alliance (NAUTICA). Int J Antimicrob Agents 2006;27:475-88.

11. Jang MK, Chu JM, Lee HS, Kim JS. Changes of antimicrobial resistance of Gram negative bacilli isolated in Chonbuk national university hospital(1993-1997). J Korean Pediatr Soc 2000;43:625-31.

12. Yoon JE, Kim WK, Lee JS, Shin K-S, Ha T-S. Antibiotic susceptibility and imaging findings of the causative microorganisms responsible for acute urinary tract infection in children: a five-year single center study. Korean J Pediatr 2011;54:79-85.
13. Yun KW. Principles of antibiotic therapy in pediatric emergency department. Pediatr Emerg Med J 2015;2:59-66.

14. Kim SH, YooRN, Lee JA. The impact of the antibiotic burden on the selection of its resistance among gram negative bacteria isolated from children. Pediatr Infect Vaccine 2015;22:178-85.

15. Hur JK. The use of fluoroquinolone in children. Korean J Pediatr 2008:51:1042-6.

16. Aihua Wang, Yonghong Yang, Quan Lu, Yi Wang, Yuan Chen, Li Deng,et al. Presence of qur gene in Escherichia coli and Klebsiella pneumoniae resistant to ciprofloxacin isolated from pediatric patients in China. BMC Infectious Diseases 2008;8:68.

17. Du Bois SK, Marriot MS, Amyes S.G. TEM- and SHV-derived extended-spectrum beta-lactamase : Relationship between selection, structure and function. J Antimicrob Chemother 1995;35:722.

18. Pai $\mathrm{H}$. The characteristics of extended-spectrum beta-lactamases in Korean isolates of Enterobacteriaceae. Yonsei Med J 1998;39: 514-9.

19. Lee JW, Shin JS, Seo JW, Lee MA, Lee SJ. Incidence and Risk Factors for Extended-Spectrum beta-Lactamase-Producing Escherichia coli in Community-acquired Childhood Urinary Tract Infection. J Korean Soc Pediatr Nephrol 2004;8:214-222.

20. Park C, Kim MS, Kim MK, Yim HE, Yoo KH, Hong YS, Lee JW. Clinical Significance of Extended-Spectrum beta-Lactamase Producing Escherichia coli in Pediatric Patients with Febrile Urinary Tract Infection. J Korean Soc Pediatr Nephrol 2012;16:38-45.

21. Ahn DH, Kim KW, Cho HK, Tchah H, Jeon IS, Ryoo E, Sun YH. Febrile Urinary Tract Infections Caused by Community-Acquired Extended-Spectrum beta-Lactamase-Producing and-Nonproducing Bacteria: A Comparative Study. Pediatr Infect Vaccine 2015;22:29-35.

22. Kim NH, Lee JA, Kim YK, Choi EH, Ha IS, Lee HJ, Choi Y. Risk Factors of Urinary Tract Infections Due to Extended-spectrum $\beta$-lactamase Producing Escherichia coli in Children. Korean J Pediatr 2004:47:164-9. 